\title{
Photoprotection-UV Filters: Properties, Use and Safety in Sun Products
}

\author{
Antonina Agolzer \\ StudioAgolzer, Studio Medico di Dermatologia, via Gorghi 5 Udine 33100, Italy
}

\begin{abstract}
Sunscreen application is a common strategy used to prevent the damage induced by UV radiations. In the last 30 years a variety of protective agents against UV exposure have been developed: physical filters, chemical filters and organic filters. Physical filters are the safest: they scatter and reflect UV rays, but are difficult to apply on the skin. Chemical filters are capable of absorbing these rays, they are cosmetically pleasant, but recent studies suggest a toxicological nature for some of these agents. Organic filters are the newest advance in sun products. Since 2003 the COLIPA Index [Europe] has regulated sun product availability on the market. In the USA, the FDA establishes the classification and the safety of sun products, which are considered topical medical drugs. This article summarizes the dermatologist's point of view, according to the patient's request, professional ethics and increasing offer of sun product.
\end{abstract}

Key words: Photoprotection, UV filters, skin cancer, skin damage, physical filters, chemical filters, organic filters.

\section{Introduction}

The sun has always been of vital importance for life on earth: it gives light, warmth and energy. Life without the sun would be impossible.

What's the sun for? The beneficial effects for humans are the production of vitamin $\mathrm{D}$, an antidepressive and therapeutical effect for the mind, the antibacterial power and a lot more. The harmful effects on the skin are erythema, photo allergy, DNA mutations, aging, carcinogenesis.

UV rays consist of many types of radiation (Fig. 1): the light that we see consist of a number of waves having various wave lengths that we can perceive thanks to a prism or with the rainbow.

However, there are some rays that we cannot see: namely: x-rays, gamma rays, ultraviolet rays, infrared, radio waves.

In the history of photoprotection there are this important dates [1]:

1801 Ritter discovered the UV rays;

1922 Hausser and Vahle connected action spectra

Corresponding author: Antonina Agolzer, medical doctor, research fields: dermatologist. of erythema and pigmentation;

1932 Coblenz divided UV into UVA, UVB and UVC.

What kinds of radiation are reaching the earth?

(Fig. 2)

UVB-UVA rays 4\%;

Visible radiation 52\%;

Infrared (IR) 44\%.

Ultraviolet UVA rays are responsible for skin pigmentation. They penetrate more forcefully, they harm our collagen or elastic fibres. On the long term they bring about the precocious aging of our skin with dryness, loss of elasticity, increase in the number of wrinkles, freckles, dilation of teleangectasia.

As soon as exposure to sun begins, the first darkening is linked to oxidation of melanin which is already present in the epidermis. If exposure continues, the synthesis of new melanin fostered, favouring the tan prolongation.

Ultraviolet UVB rays have a little penetration power, they stimulate melanin synthesis and provoke DNA cell damage, which is immediately repaired, but on the long term they can cause skin tumors (photo carcinogenesis). After 2-6 hours from exposure they 


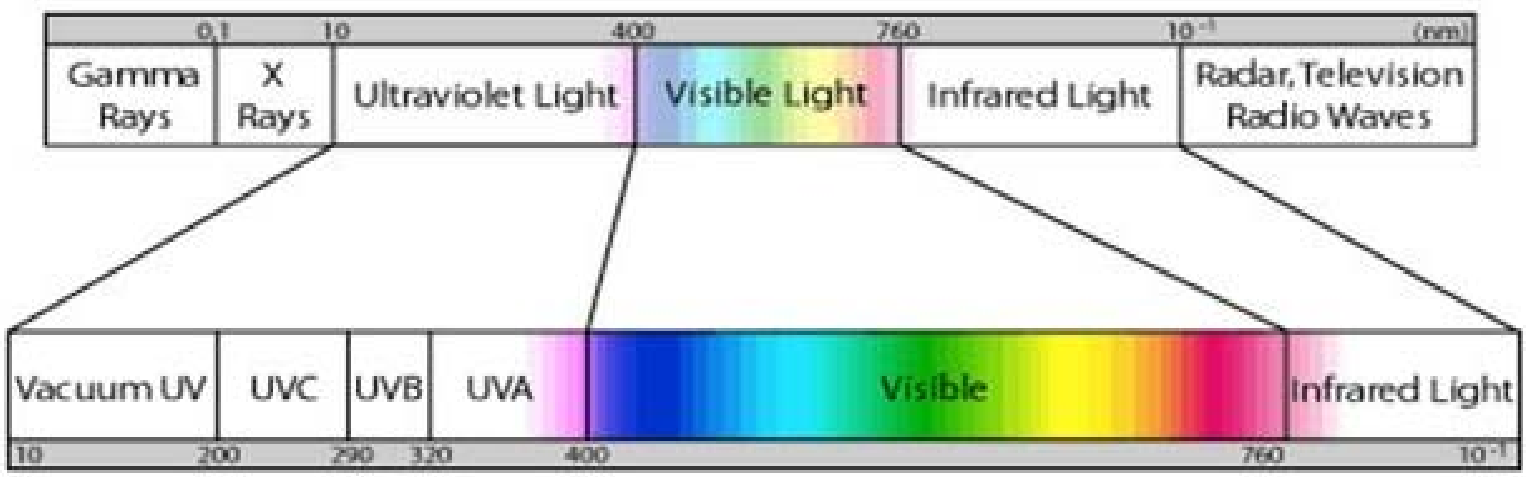

\section{UV RAYS CONSIST OF MANY TYPES OF RADIATION}

Fig. 1 UV rays consist of many types of radiation.

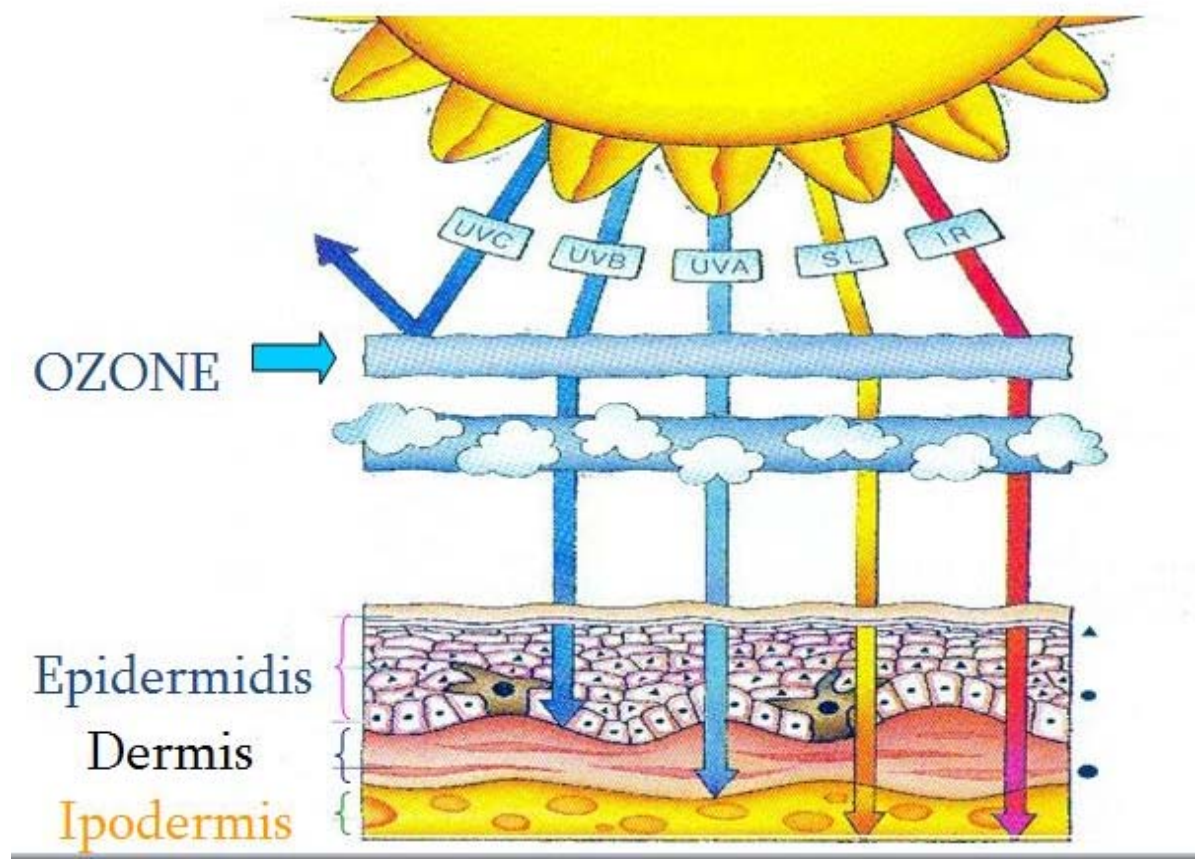

Fig. 2 What kinds of radiation are reaching the earth?

provoke an inflammatory response of the skin with more or less intense redness. After 2-3 days, if exposure to sun continues, a tan comes about which reaches its maximum intensity in 7-10 days.

The pathogenesis of the UVA-UVB rays-mediated damage is shown in Fig. 3.

\section{Topical Photoprotection: Current Status}

Sunscreen application is the main strategy used to prevent the problems caused by short or chronic exposition to the sun. Skin cancer incidence is continuously increasing: sun exposure has been indicated as a primary cause of such a growth. The phenomenon is particularly pronounced among Caucasians living in developed countries.

Are some changes in lifestyle the main trigger? Most people consider suntan a sign of physical and social well-being. The use of artificial tanning devices that emits UVA-UVB radiation has become increasingly popular among young people in Europe.

The implementation of cutaneous photo protection appears more feasible nowadays and in the immediate 


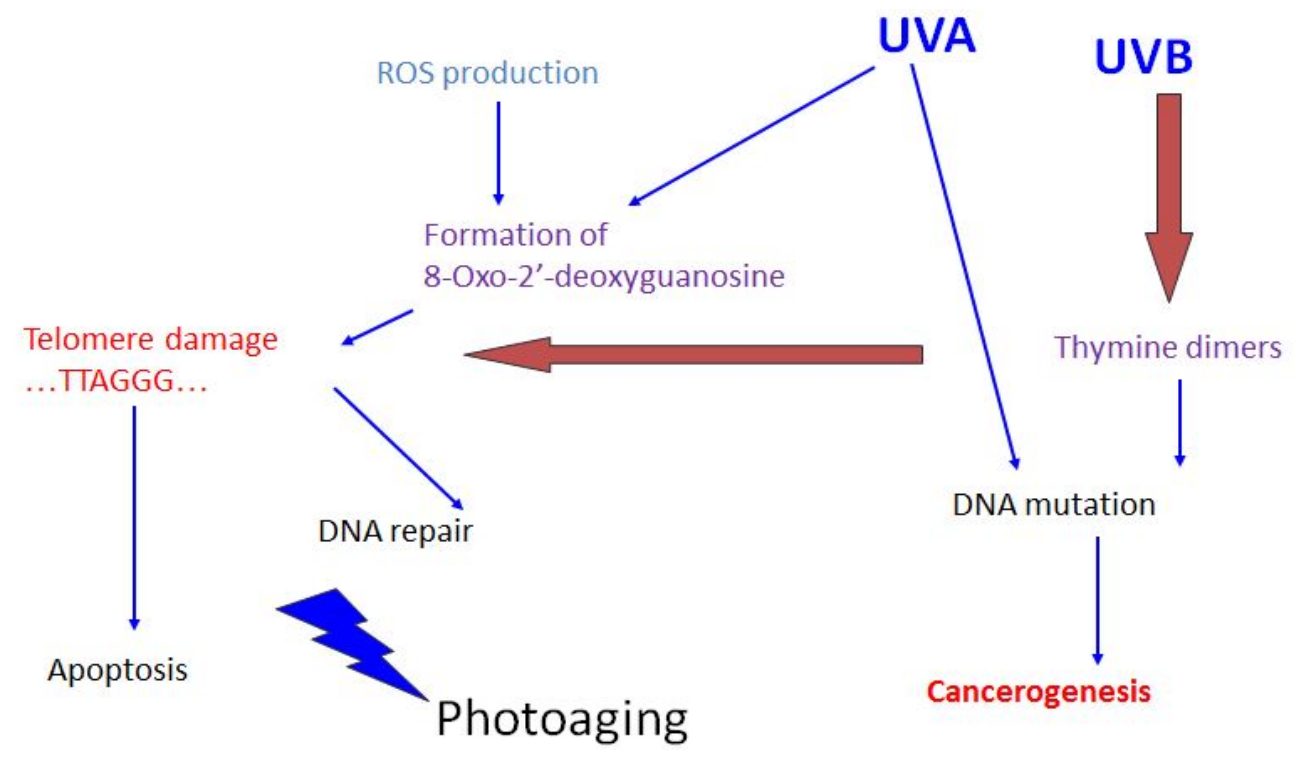

Fig. 3 The pathogenesis of the UVA-UVB rays-mediated damage.

future the large use of products that are able to neutralize UV radiation has been advocated by many institutions as the most appropriate means to reduce skin damage from solar radiation: photoaging and photocarcinogenesis.

A radical change can be obtained through education as to the correct way of enjoying appropriate sun exposure during all one's life.

Sunscreen prevents sunburn and protects skin cells (including melanocytes) from mutations induced by ultraviolet radiations. There are many prospective cohort studies and only one randomized trial about this question [2]. These studies suggest that regular sunscreen use reduces risk of melanoma, but this behavior is uncommon, even in populations residing in areas of high sunlight levels. Under plausible scenarios of increasing prevalence of sunscreen use, cumulative incidence of melanoma between 2012 and 2031 could reduce approximatively $10 \%$ in high incidence of populations (respectively 231.053 fewer melanomas would arise in the U.S. white population and 28.071 fewer melanomas would arise in Australia) [2].

The only randomized controlled trial of sunscreen for melanoma prevention, the Nambour Trial [3] was designed to evaluate basal and squamous cell carcinoma and their precursors, with melanoma as a secondary end point. It showed a none statistically significant protective effect for all melanoma (11 melanomas in the sunscreen group vs. 22 in the control group) and a statistically significant protective effect for invasive melanoma (3 invasive melanoma in the sunscreen group vs. 11 in the control group).

This study is the best available evidence, but has limitation, such as the small number of melanoma diagnosed during the study [4].

Suntan is a cutaneous reaction against the UV rays: the production of melanin needs more time that people think. Melanin is synthetized by a few cells in the epidermis, melanocytes shaped as granules, which later transfer into the keratinocytes, protecting their nucleus from the action of UVs.

Melanin is produced by oxidation of the aminoacid tyrosine, followed by polymerization. There are three basic types of melanin: eumelanin, pheomelanin and neuromelanin. The most common type is EUMELANIN, of which there are two types: brown eumelanin and black eumelanin. PHEOMELANIN is a cysteine that contains red polymer of benzothiazine units largely responsible for red hair. 
NEUROMELANIN is found in the brain, though its function remains obscure. In human skin, melanogenesis is initiated by exposure to UV radiation, causing the skin to darken. Melanin is an effective absorbent of light; the pigment is able to dissipate over $99.9 \%$ of absorbed UV radiation.

Therefore tanning represents a self defence response, a skin protection linked to the production of melanic pigment and to cutaneous thickening, which brings about a pigmentation far superior compared to the genetic one of the individual.

The PHOTOTYPE indicates the way our skin reacts to sun rays: identifying one's own phototype is important to expose oneself to the sun in a safer way (Fig. 4).

Phototype 1 is characterized by red hair, blue eyes, extremely fair skin. It always gets burned and never tans. This skin has very few natural defences from the sun, therefore these people must be extremely careful with our protection: T-shirt, headgear, sunglasses, beach umbrella and utmost protection solar cream. These people always need an extremely high photoprotection. Phototype 2 is characterized by blond hair, blue or green eyes, fair skin. It always gets burned and tans little. They must expose to the sun carefully and gradually, always using products with extremely high photoprotection. Phototype 3 shows chestnut, brown hair and brown-green eyes, fair skin.

It gets moderately burned and tans gradually. It is necessary to use a high photoprotection solar product during the first days of exposure, after that the protection factor can be reduced. People with phototype 4 present dark brown hair and eyes, olive-greenish skin. It gets burned very little and always tans, and fast. I should also remember phototype 5 (dark hair eyes and skin, it rarely gets burned, it tans intensely and easily) and phototype 6 (black hair, eyes and skin: it never gets burned, it is always intensely pigmented).

In the history of photoprotection we can distinguish

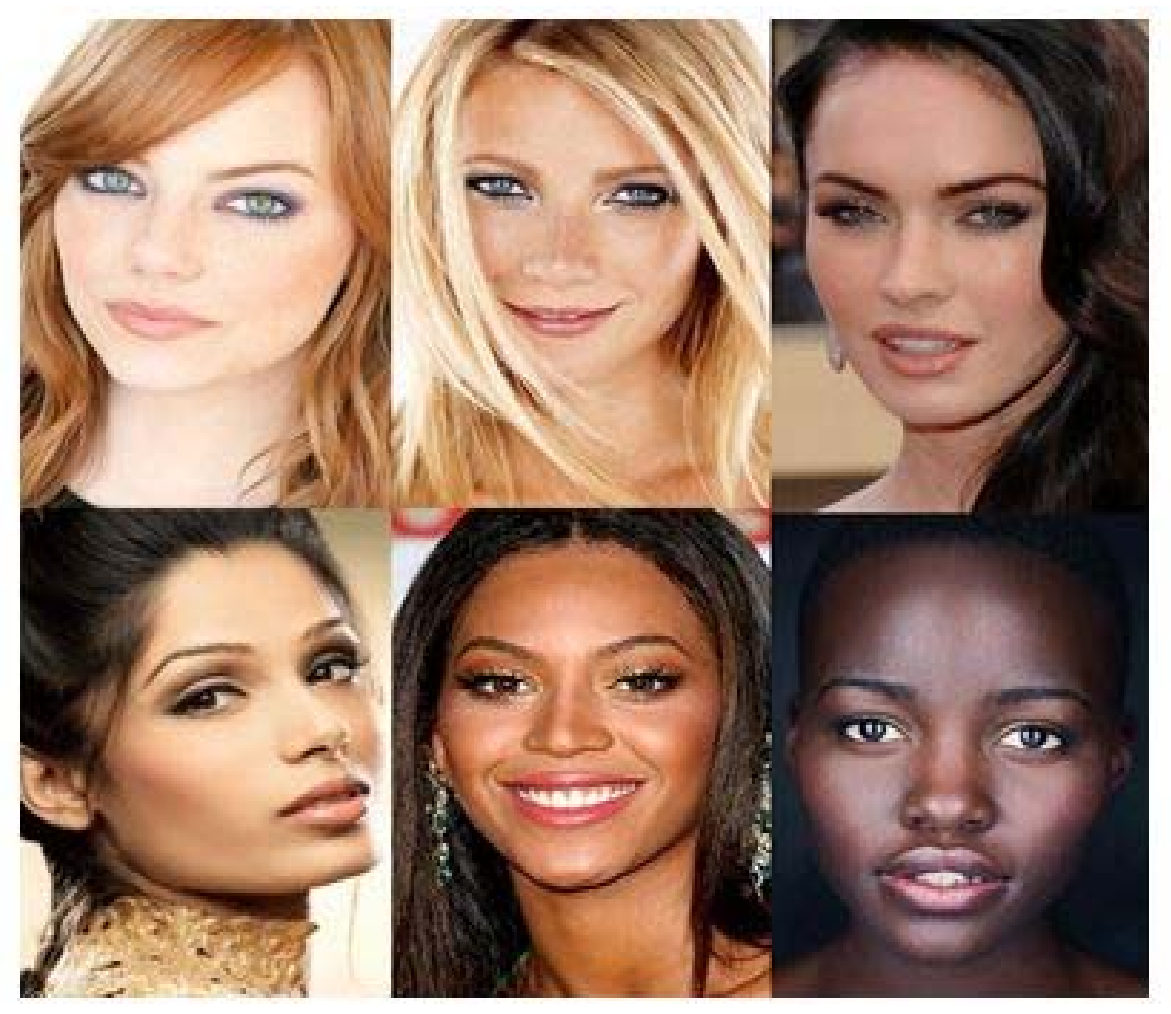

Fig. 4 The РНОTОTYPE indicates the way our skin reacts to sun rays: identifying one's own phototype is important to expose oneself to the sun in a safer way. 
some important dates, when the following products became available [1]:

1928 the first sunscreen (benzyl salicylate and benzyl cinnamate) in the USA;

1948 PABA esters;

1989 micronized inorganic filter (titanium dioxide);

1992 micronized inorganic filter (zinc oxide).

Up to 10 years ago dermatologist only had physical and some chemical filters available as recommended photoprotective agents; nowadays specialist can also advise the use of new organic chemical filters.

The advantages of PHYSICAL FILTERS are: uniform barrier against UVBs, UVAs, IR, because they act as physical mirrors. They provide good resistance to water, they are characterized by good photostability (no chemical reaction), no risks of contact dermatitis or photo allergies.

On the other hand, the disadvantages of PHYSICAL FILTERS are: low cosmetic quality, whitish because they reflect radiation, the user needs big quantities because they are difficult to apply.

In the last few years research has been able to micronize physical filters in metal particles to a size of 100-150 nm. This way, cosmetically they are more appreciated, but their filter capacity is reduced. Furthermore we should consider the risk of absorption through skin. In the last two years some articles discussed the relationship between sunscreen, make-up, moisturizers containing physical filters and frontal fibrosing alopecia [5, 6]. This pathological condition of the scalp is more frequent in female and in postmenopausal age. Traces of titanium dioxide in the hair follicle are the suggested cause for the lichenoid reaction in fibrosing alopecia [7].

Chemical filters, which are cosmetically pleasant, have the following disadvantages: action mediated by chemical reaction, limited absorption spectra, photoallergy risk, variable resistance to water. The photostability of chemical filters is very different. Octocrylene shows photostability; but contact and photocontact allergy are reported [8]. Chemical filters with limited photostability can produce toxic photoproducts, the emission of light with a larger wave length is possible. It is debated whether there is a skin tumor risk and other unwanted side effect with this type of chemical filters [9]. It is important to remember a contemporary review [10] regarding the neurotoxicity of several organic chemical filters and two allowed physical filters (zinc oxide and titanium dioxide). Dermal exposure is the most relevant entry route of chemicals related to sunscreen use, but gastrointestinal and pulmonary exposure should also be considered during eating and drinking with sunscreen applied on hands and lips [10]. Swimming in contaminated water or drinking water with trace of sun products might increase the contact (environmental exposure). We should also consider the environmental pollution given by sunscreen applied during bathing and the contact with fish, crustaceans and the coral reef [10]. In this review the authors inform about the maximum allowed concentration for every filter in US and Europe, e.g. for octocrylene the maximum concentration allowed is $10 \%$. The effects of repeated, long-term, low doses exposures to single compounds and mixtures of various UV filters are also poorly studied.

In the last years research has shown that the synergy between more chemical filters enhances UVB and UVA protection, with chemical complexes which give uniformity of protection and more photostability.

In fact the aim of a good photoprotection is a large and stable cover against UVB and UVA. The majority of chemical filters give protection only against UVB rays (Fig. 5).

It is important to inform the users, that they should always pay attention to the effective protection reported on the package against the UVB and UVA.

The future photoprotection target is the cover against the visible spectra and against the infrared radiation (Figs. 1 and 2), because these are responsible of the chronic photoaging. Specifically infrared radiation caused increased expression of MMP-1 in the 


\begin{tabular}{|c|c|c|c|}
\hline \multirow[t]{2}{*}{$\begin{array}{l}\text { FDA Monograph } \\
\text { Sunscreen Ingredients }\end{array}$} & \multicolumn{2}{|c|}{$\begin{array}{l}\text { Amount of } \\
\text { Ray Protection }\end{array}$} & \multirow[t]{2}{*}{$\begin{array}{l}\text { Chemical (C) } \\
\text { or Physical (P) }\end{array}$} \\
\hline & UVA & UVB & \\
\hline Aminobenzoic acid (PABA) & 0 & - & C \\
\hline Avobenzone & - & 0 & $\mathrm{C}$ \\
\hline Cinoxate & 0 & (2) & C \\
\hline Dioxybenzone & D & - & C \\
\hline Ecamsule & ? & () & C \\
\hline Homosalate & 0 & ? & C \\
\hline Menthyl anthranilate & D & ? & C \\
\hline Octocrylene & ()) & ? & C \\
\hline Octyl methoxycinnamate & O & ? & C \\
\hline Octyl salicylate & 0 & ? & C \\
\hline Oxybenzone & (1) & ? & c \\
\hline Padimate $\mathrm{O}$ & 0 & ? & C \\
\hline Phenylbenzimidazole & $\mathrm{O}$ & ? & C \\
\hline Sulisobenzone & D & (1) & C \\
\hline Titanium dioxide & D & - & $P$ \\
\hline Trolamine salicylate & 0 & ? & C \\
\hline Zinc Oxide & ? & ? & $P$ \\
\hline
\end{tabular}

Fig. 5 The majority of chemical filters gives protection only against UVB rays.

dermis, but not in the epidermis [11].

The fractioned biomimetic melanin is a new protection agent, which absorbs the wave length from 400 to $500 \mathrm{~nm}$ : it is derivated from natural tyrosine of vegetable origin [12].

The presence of antioxidants agents in the sunscreen products is very important: oxothiazolidine (OTZ10), polypodium leucotomos (PLE), ferulic acid, vitamin $\mathrm{C}$ (ascorbic acid), vitamin $\mathrm{E}$ acetate, green tea, thermal water.

The new concept is an active photoprotection, with the cover against UVA-UVB combined with liposome-containing DNA repair enzymes against clinical evidence of sun damage (e.g. actinic keratosis): oxoguanine glycosylase (OGG-1), photolyase, endonuclease [13].

Last but not least, it is important to discuss about the SPF (sun protection factor). Introduced in 1974, it measures the fraction of sunburn producing UV rays that reach the skin. If a person develops a sunburn in 10 min, when not wearing a sunscreen, the same person in same intensity of sunlight will avoid sunburn for 200 min if wearing a sunscreen with an SPF of 20. SPF 20 means that $1 / 20$ of the burning radiation will reach the skin, assuming sunscreen is applied evenly at a thick dosage of 2 milligrams per square centimeter $\left(\mathrm{mgr} / \mathrm{cm}^{2}\right)$. The definition of the SPF is multiplying the SPF factor by the length of time it takes from him or her to suffer a burn without sunscreen. It is important to consider that the right dose for one adult is approximately 30-40 grams every application and the right time for reapplication is every 2 hours.

When the SPF is very high, people stay under the 
sun more time and longer, during the dangerous hours (11 AM to $3 \mathrm{PM}$ ), apply fewer products than that is needed and do not use physical protection like headgear, sunglasses, umbrella [14].

The use of a higher sunscreen than needed was recently suggested in one commentary published on the blue journal of AAD [15], because many studies have shown that people will routinely apply to the skin only one-third to one-half of the amount of sunscreen that the US Food and Drug administration requires during testing for assigning an SPF to sunscreens. In fact the application of 0.5 milligrams per square centimeter (only one quarter of the right dose) reduces by $75 \%$ the original SPF.

Finally it is important to remember the correct behavior during sun exposure: the solar product must be applied $30 \mathrm{~min}$ before exposure to the sun and the reapplication is necessary every 2 hours, after bathing and after profuse sweating. People must avoid the warmer times of the day: from $11 \mathrm{AM}$ to $4 \mathrm{PM}$ the rays are direct and aggressive (50\% of UV rays are emitted from 11 AM to $3 \mathrm{PM}$ ). The exposure must be gradual and progressive, the protection of the eyes with sunglasses with certified protection UVA-UVB is necessary. It is important to know that up to $90 \%$ UV rays penetrate through the clouds, the wind increases the chances of sunburn (it decreases the perception of heat), reflective surfaces (snow and sand) increase the exposure to UV rays. In the water we receive $40-95 \%$ of radiation, under the umbrella we receive $50 \%$ of radiation and in the mountains the radiations are more powerful. Parents must avoid sun exposure for the new-born and keep it limited for children. Physicians must recommend their patients to avoid sun exposure after chemical peeling or recent scars (post surgery or post traumatic).

Avoidance of photosensitizing chemical substances application during sun exposure should also be recommended: fragrance, perfume, deodorant can enhance the radiation damage. The dermatologist knows a long list of topical photosensitizing drug and substances: benzoyl peroxide, clindamycin, retinoids, alfa and beta hydroxiacids, antihistamines (desclopheniramine), tacrolimus, ketoprofen, bergamot and henna, salicylic acid etc. There are also a long list of phototoxic and photosensitizing drugs for systemic use: antibiotics (tetracycline, levofloxacin, isoniazide, sulfametossazole), antimycotics (griseofulvin e ketitofen), antineoplastic agents (florouracil, paclitaxel), targeted therapies (imatinib, erlotinib, verumafenib etc.), interferon, methotrexate, diclofenac, ibuprofen, atenolol, hydrochlorothiazide, allopurinol, acyclovir, oral retinoids, fluoxetine, omeprazole, ranitidine etc.

\section{Conclusions}

Suntan is a cutaneous reaction against UV rays and production of melanin needs more time than people think. The implementation of cutaneous photoprotection appears more feasible nowadays and in the immediate future. A radical change can be obtained through education as to the correct way of enjoying appropriate sun exposure during all one's life. Physicians must recommend a safe and conscious behavior against the sun. Public health organization should consider novel sun protection strategies such as woven clothing and oral photoprotection. On the other side consumers should prefer safe types of sunscreen, by choosing chemist shop of pharmacies and personal retailer: the composition is always declared (INCI in Europe), but not the percentage for every (chemical) filter. Research in the future should foster the discovery of new, alternative, safer and more efficient UV filters.

\section{Acknowlegments}

The author gratefully acknowledge Prof. Giuseppe Stinco Head of Dermatology Institute, Department of Experimental and Clinical Medicine, University of Udine Italy and Prof. Valentina Rapozzi, Department of Medical and Biological Sciences, University of Udine for the invitation to the” Photodermatology 
Symposium" (Udine, June 22 ${ }^{\text {nd }}$, 2018-International ALPE-ADRIA Meeting on Photobiology-Udine 20-22 June 2018 ) to speak about this issue.

\section{References}

[1] Lim, W. L., and Draelos, Z. D. 2009. Clinical Guide to Sunscreen and Photo Protection. New York, USA: Informa Healthcare Inc.

[2] Olsen, C. M., Wilson, L. F., Green, A. C., Biswas, N., Lovalka, J., and Whiteman, D. C. 2018. "How Many Melanomas might Be Prevented if More People Applied Sunscreen Regularly?” British Journal of Dermatology 178 (1): 140-7.

[3] Green, A. C., Williams, G. M., Logan, V., and Strutton, G. M. 2011. "Reduced Melanoma after Regular Sunscreen Use: Randomized Trial Follow-up.” Journal Clin Oncol 29 (3): 257-63.

[4] Wehner, M. R. 2018. "Sunscreen and Melanoma Prevention: Evidence and Expectation.” British Journal of Dermatology 178 (1): 15-6.

[5] Donati, A. 2016. "Frontal Fibrosing Alopecia and Sunscreens: Cause or Consequence?” British Journal of Dermatology 175 (4): 675-6.

[6] Debroy Kidambi, A. et al 2017. "Frontal Fibrosing Alopecia in Men: An Association with Facial Moisturizers and Sunscreens.” British Journal of Dermatology 177 (1): 260-1.

[7] Gary, C. et al. 2017. “Alopècie frontale fibrosante post-mènopausique: une rèaction lichenoide aux nanoparticules de dioxyde de titane prèsentes dans les follicules pileux?” Annales de Dermatologie et de Vènèrèologie 144 (12): 206.

[8] de Groot, A. C., and Roberts, D. W. 2014. "Contact and Photocontact Allergy to Octocrylene: A Review.” Contact Dermatitis 70 (1): 193-204.

[9] Bora, N. S., Mazumder, B., and Chattopadhyay, P. 2018. "Prospects of Topical Protection from Ultraviolet Radiation Exposure: A Critical Review on the Juxtaposition of the Benefits and Risks Involved with Use of Chemoprotective Agents.” J Dermatolog Treat 29 (1): 256-68.

[10] Ruszkiewicz, J. A., Pinkas, A., Ferrer, B., Peres, T. V., Tsatakis, A, and Ashner, M. 2017. "Neurotoxic Effect of Active Ingredients in Sunscreen Products, a Contemporary Review.” Toxicology Reports 4: 245-59.

[11] Schroeder, P. et al. 2008 "Infrared Radiation-Induced Matrix Metalloproteinase in Human Skin: Implications for Protection.” J invest Dermatol 128 (10): 2491-7.

[12] Vij, M. et al. 2016 "Bioinspired Functionalized Melanin Nanovariants with Range of Properties Provide Effective Color Matched Photoprotection in Skin.” Biomacromolecules 17 (9): 2912-9.

[13] Megna, M., Lembo, S., Balato, N., and Monfrecola, G. 2017 “'Active”” Photoprotection: Sunscreens with DNA Repair Enzymes.” Giorn. Ital. Dermatol. Venerol. 152 (1): 302-7.

[14] Auler, P. 2009. "Sunscreen Abuse for Intentional Sun Exposure” British Journal of Dermatology 161 (suppl 3): 40-5.

[15] Linden, K. G. 2018 "Sun Protection Factor [SPF]: Is Higher Better?” Journal of Am Acad of Dermatology published on line: March 13, 2018. 